\title{
On the singularity and distribution parameters of involutive and Bertrandian Frenet ruled surfaces in $\mathbb{E}^{3}$
}

\author{
Seyda Kilicoglu ${ }^{1}$ and Suleyman Senyurt ${ }^{2}$ \\ ${ }^{1}$ Faculty of Education, Department of Mathematics, Başkent University, Ankara, Turkey \\ ${ }^{2}$ Faculty of Arts and Sciences, Department of Mathematics, Ordu University, Ordu, Turkey
}

Received: 30 December 2015, Accepted: 6 February 2016

Published online: 22 June 2016.

\begin{abstract}
In this paper we consider twelve special ruled surfaces associated to the curve $\alpha$,the involute curve $\alpha^{*}$ and Bertrand mate $\alpha^{* *}$, with $k_{1} \neq 0$. They are called as Frenet ruled, involutive Frenet ruled and Bertrandian Frenet ruled surfaces, cause of their generators are the Frenet vector fields of curve $\alpha$. First we give all the parametrizations of all Frenet ruled surfaces in terms of the Frenet apparatus of curve $\alpha$. We examined distribution parameters and Singularity of involutive and Bertrand Frenet ruled surfaces based on the Frenet apparatus of curve $\alpha$ in $\mathrm{E}^{3}$.
\end{abstract}

Keywords: Involute curve, Bertrand curve pair, striction curves, ruled surfaces, Frenet ruled surface.

\section{Introduction and preliminaries}

A ruled surface can always be described (at least locally) as the set of points swept by a moving straight line. A ruled surface is one which can be generated by the motion of a straight line in Euclidean 3-space [2]. Choosing a directrix on the surface, i.e. a smooth unit speed curve $\alpha(s)$ orthogonal to the straight lines, and then choosing $v(s)$ to be unit vectors along the curve in the direction of the lines, the velocity vector $\alpha_{s}$ and $v$ satisfy $\left\langle\alpha^{\prime}, v\right\rangle=0$. The striction point on a ruled surface $\varphi(s, v)=\alpha(s)+v X(s)$ is the foot of the common normal between two consecutive generators (or ruling). Deriving curves based on the other curves is a subject in geometry. Involute-evolute curves, Bertrand curves are these kind of curves. The involute of a given curve is a well-known concept in $\mathbb{I R}^{3}$, [1]. We can say that evolute and involute is a method of deriving a new curve based on a given curve. Let $\alpha$ and $\alpha^{*}$ be the curves in Euclidean $3-$ space. The tangent lines to a curve $\alpha$ generate a surface called the tangent surface of $\alpha$. If the curve $\alpha^{*}$ which lies on the tores intersect the tangent lines orthogonally is called an involute of $\alpha$. If a curve $\alpha^{*}$ is an involute of $\alpha$, then by definition $\alpha$ is an evolute of $\alpha^{*}$. Hence given $\alpha$, its evolutes are the curves whose tangent lines intersect $\alpha$ orthogonally. By using the similiar method we produce a new ruled surface based on the other ruled surface. The differential geometric elements of the involute $\tilde{D}$ scroll are examined in [9]. It is well-known that, if a curve is differentiable in an open interval, at each point, a set of mutually orthogonal unit vectors can be constructed. And these vectors are called Frenet frame or moving frame vectors. The rates of these frame vectors along the curve define curvatures of the curves. The set, whose elements are frame vectors and curvatures of a curve $\alpha$, is called Frenet-Serret apparatus of the curves. Let Frenet vector fields be $V_{1}(s), V_{2}(s), V_{3}(s)$ of $\alpha$ and let the first and second curvatures of the curve $\alpha(s)$ be $k_{1}(s)$ and $k_{2}(s)$, respectively. The quantities $\left\{V_{1}, V_{2}, V_{3}, D, k_{1}, k_{2}\right\}$ are collectively Frenet-Serret apparatus of the curves. Let a rigid object move along a regular curve described parametrically by $\alpha(s)$. For any unit speed curve $\alpha$,in terms of the Frenet-Serret apparatus, the Darboux vector can be expressed as

$$
D(s)=k_{2}(s) V_{1}(s)+k_{1}(s) V_{3}(s),[3] .
$$


where curvature functions are defined by $k_{1}=k_{1}(s)=\left\|V_{1}(s)\right\|$ and $k_{2}(s)=-\left\langle V_{2}, \dot{V}_{3}\right\rangle$. Let a vector field be

$$
\tilde{D}(s)=\frac{k_{2}}{k_{1}}(s) V_{1}(s)+V_{3}(s)
$$

along $\alpha(s)$ under the condition that $k_{1}(s) \neq 0$ and it is called the modified Darboux vector field of $\alpha$ [7]. Let unit speed regular curve $\alpha: I \rightarrow \mathbb{E}^{3}$ and $\alpha^{*}: I \rightarrow \mathbb{E}^{3}$ be given. For $\forall s \in I$, then the curve $\alpha^{*}$ is called the involute of the curve $\alpha$, if the tangent at the point $\alpha(s)$ to the curve $\alpha$ passes through the tangent at the point $\alpha^{*}(s)$ to the curve $\alpha^{*}$. The distance between corresponding points of the involute curve in $\mathbb{E}^{3}$ is

$$
d\left(\alpha(s), \alpha^{*}(s)\right)=|c-s|, c=\text { constant }, \forall s \in I
$$

then we may write that

$$
\alpha^{*}(s)=\alpha(s)+(c-s) V_{1}(s),[4]
$$

Let $\alpha: I \rightarrow \mathbb{E}^{3}$ and $\alpha^{*}: I \rightarrow \mathbb{E}^{3}$ be the $C^{2}$ - class differentiable unit speed two curves and the quantities $\left\{V_{1}, V_{2}, V_{3}, \tilde{D}, k_{1}, k_{2}\right\}$ and $\left\{V_{1}^{*}, V_{2}^{*}, V_{3}^{*}, \tilde{D}^{*}, k_{1}^{*}, k_{2}^{*}\right\}$ are collectively Frenet-Serret apparatus of the curves $\alpha$ and the involute $\alpha^{*}$, respectively, then

$$
\left\{\begin{array}{l}
V_{1}^{*}=V_{2}, \\
V_{2}^{*}=\frac{-k_{1}}{\left(k_{1}^{2}+k_{2}^{2}\right)^{\frac{1}{2}}} V_{1}+\frac{k_{2}}{\left(k_{1}^{2}+k_{2}^{2}\right)^{\frac{1}{2}}} V_{3} \\
V_{3}^{*}=\frac{k_{2}}{\left(k_{1}^{2}+k_{2}^{2}\right)^{\frac{1}{2}}} V_{1}+\frac{k_{1}}{\left(k_{1}^{2}+k_{2}^{2}\right)^{\frac{1}{2}}} V_{3}
\end{array}\right.
$$

and

$$
\tilde{D}^{*}=\frac{k_{2}}{\left(k_{1}^{2}+k_{2}^{2}\right)^{\frac{1}{2}}} V_{1}-\frac{k_{1}^{\prime} k_{2}-k_{1} k_{2}^{\prime}}{\left(k_{1}^{2}+k_{2}^{2}\right)^{\frac{3}{2}}} V_{2}+\frac{k_{1}}{\left(k_{1}^{2}+k_{2}^{2}\right)^{\frac{1}{2}}} V_{3}
$$

the first curvature and second curvature of involute $\alpha^{*}$ are given by

$$
k_{1}^{*}=\frac{\sqrt{k_{1}^{2}+k_{2}^{2}}}{(c-s) k_{1}}, k_{2}^{*}=\frac{-k_{2}^{2}\left(\frac{k_{1}}{k_{2}}\right)^{\prime}}{(c-s) k_{1}\left(k_{1}^{2}+k_{2}^{2}\right)},[4] .
$$

Let $\alpha: I \rightarrow \mathbb{E}^{3}$ and $\alpha^{* *}: I \rightarrow \mathbb{E}^{3}$ be the $C^{2}$ - class differentiable unit speed two curves and let $V_{1}(s), V_{2}(s), V_{3}(s)$ and $V_{1}^{* *}(s), V_{2}^{* *}(s), V_{3}^{* *}(s)$ be the Frenet frames of the curves $\alpha$ and $\alpha^{* *}$, respectively. If the principal normal vector $V_{2}$ of the curve $\alpha$ is linearly dependent on the principal normal vector $V_{2}^{* *}$ of the curve $\alpha^{* *}$, then the pair $\left(\alpha, \alpha^{* *}\right)$ are called Bertrand curve pair, [4,8]. Also $\alpha^{* *}$ is called Bertrand mate. If the curve $\alpha^{* *}$ is Bertrand mate of $\alpha$, then we may write that

$$
\alpha^{* *}(s)=\alpha(s)+\lambda V_{2}(s)
$$

If the curve $\alpha^{* *}$ is Bertrand mate $\alpha(s)$, then we have that $\left\langle V_{1}^{* *}(s), V_{1}(s)\right\rangle=\cos \theta=$ constant. The distance between corresponding points of the Bertrand curve pair in $\mathbb{E}^{3}$ is constant, $[4,8] . \alpha(s)$ is a Bertrand curve if and only if there exist nonzero real numbers $\lambda$ and $\beta$ such that constant $\lambda k_{1}+\beta k_{2}=1$ for any $s \in I$. It follows from this fact that a circular helix is a Bertrand curve, [4]. Frenet-Serret apparatus of the curves $\alpha$ and the Bertrand mate $\alpha^{* *}$ as follows,

$$
\left\{\begin{array}{l}
V_{1}^{* *}=\frac{\beta}{\sqrt{\lambda^{2}+\beta^{2}}} V_{1}+\frac{\lambda}{\sqrt{\lambda^{2}+\beta^{2}}} V_{3} \\
V_{2}^{* *}=V_{2} \\
V_{3}^{* *}=\frac{-\lambda}{\sqrt{\lambda^{2}+\beta^{2}}} V_{1}+\frac{\beta}{\sqrt{\lambda^{2}+\beta^{2}}} V_{3}
\end{array}\right.
$$


and

$$
\tilde{D}^{* *}=\frac{\sqrt{\lambda^{2}+\beta^{2}}}{\left(\beta k_{1}-\lambda k_{2}\right)}\left(k_{2} V_{1}+k_{1} V_{3}\right) .
$$

the first and second curvatures of the offset curve $\alpha^{* *}$ are given by

$$
\left\{\begin{array}{l}
k_{1}^{* *}=\frac{\beta k_{1}-\lambda k_{2}}{\left(\lambda^{2}+\beta^{2}\right) k_{2}}=\frac{k_{1}-\lambda\left(k_{1}^{2}+k_{2}^{2}\right)}{\left(\lambda^{2}+\beta^{2}\right) k_{2}^{2}}, \\
k_{2}^{* *}=\frac{1}{\left(\lambda^{2}+\beta^{2}\right) k_{2}},[8] .
\end{array}\right.
$$

\section{Singularity and distribution parameters of involutive Frenet ruled surfaces}

In this subsection first, we give the tangent, normal, binormal, Darboux Frenet ruled surfaces of the involute-evolute curves $\alpha^{*}$. Further we write their parametric equations in terms of the Frenet apparatus of the involute-evolute curve curve $\alpha$. Hence they are called collectively "Involutive Frenet ruled surfaces of curve $\alpha$." as in the following way. Let $\alpha: I \rightarrow \mathbb{E}^{3}$ and $\alpha^{*}: I \rightarrow \mathbb{E}^{3}$ be the $C^{2}$ - class differentiable unit speed two curves and the quantities $\left\{V_{1}, V_{2}, V_{3}, \tilde{D}\right\}$ and $\left\{V_{1}^{*}, V_{2}^{*}, V_{3}^{*}, \tilde{D}^{*}\right\}$ are collectively Frenet-Serret apparatus of the curves $\alpha$ and the involute $\alpha^{*}$, respectively,

$\varphi_{1}^{*}\left(s, v_{1}\right)=\alpha(s)+(c-s) V_{1}(s)+v_{1} V_{2}(s)$,

$\varphi_{2}^{*}\left(s, v_{2}\right)=\alpha(s)+(c-s) V_{1}(s)+v_{2}\left(\frac{-k_{1} V_{1}+k_{2} V_{3}}{\left(k_{1}^{2}+k_{2}^{2}\right)^{\frac{1}{2}}}\right)$,

$\varphi_{3}^{*}\left(s, v_{3}\right)=\alpha(s)+(c-s) V_{1}(s)+v_{3}\left(\frac{k_{2} V_{1}+k_{1} V_{3}}{\left(k_{1}^{2}+k_{2}^{2}\right)^{\frac{1}{2}}}\right)$,

$\varphi_{4}^{*}\left(s, v_{4}\right)=\alpha(s)+(c-s) V_{1}(s)+v_{4}\left(\frac{k_{2}}{\sqrt{k_{1}^{2}+k_{2}^{2}}} V_{1}-\frac{k_{1}^{\prime} k_{2}-k_{1} k_{2}^{\prime}}{\left(k_{1}^{2}+k_{2}^{2}\right)^{\frac{3}{2}}} V_{2}+\frac{k_{1} V_{3}}{\sqrt{k_{1}^{2}+k_{2}^{2}}}\right)$

are the parametrization of the ruled surface which are called involutive tangent ruled surface, involutive normal ruled surface, involutive binormal ruled surface and involutive Darboux ruled surface, respectively, [5]. Normal vector fields $\eta_{1}^{*}, \eta_{2}^{*}, \eta_{3}^{*}$, and $\eta_{4}^{*}$ of ruled surfaces $\varphi_{1}^{*}, \varphi_{2}^{*}, \varphi_{2}^{*}$, and $\varphi_{4}^{*}$ respectively, along the curve involute $\alpha^{*}$, can be expressed by the following matrix, [6]

$$
\left[\begin{array}{c}
\eta_{1}^{*} \\
\eta_{2}^{*} \\
\eta_{3}^{*} \\
\eta_{4}^{*}
\end{array}\right]=\left[\begin{array}{ccc}
0 & 0 & -1 \\
\frac{-v_{2} k_{2}^{*}}{\sqrt{\left(v_{2} k_{2}^{*}\right)^{2}+\left(1-v_{2} k_{1}^{*}\right)^{2}}} & 0 & \frac{\left(1-v_{2} k_{1}^{*}\right)}{\sqrt{\left(v_{2} k_{2}^{*}\right)^{2}+\left(1-v_{2} k_{1}^{*}\right)^{2}}} \\
\frac{-v_{2}^{*}}{\sqrt{\left(v_{3} k_{2}^{*}\right)^{2}+1}} & \frac{-1}{\sqrt{\left(v_{3} k_{2}^{*}\right)^{2}+1}} & 0 \\
0 & -1 & 0
\end{array}\right]\left[\begin{array}{c}
V_{1}^{*} \\
V_{2}^{*} \\
V_{3}^{*}
\end{array}\right]
$$

Theorem 1. Let $\varphi_{1}^{*}, \varphi_{2}^{*}, \varphi_{3}^{*}$, and $\varphi_{4}^{*}$ be involutive Frenet ruled surfaces, respectively, along the curve $\alpha$. Only involutive normal ruled surface has singular points if the curve $\alpha$ is a general helix with parameter $v_{2}=\frac{1}{k_{1}^{*}}$.

Proof. Let $\eta_{1}^{*}, \eta_{2}^{*}, \eta_{3}^{*}$, and $\eta_{4}^{*}$ be the normal vector fields of involutive Frenet ruled surfaces $\varphi_{1}^{*}, \varphi_{2}^{*}, \varphi_{3}^{*}$, and $\varphi_{4}^{*}$, respectively, along the curve $\alpha$. Using the above theorem involutive tangent ruled surface has no singular points since $V_{3}^{*}$ $\neq 0$.Involutive Darboux ruled surface has no singular points since $V_{2}^{*} \neq 0$. Involutive binormal ruled surface has no singular points under the condition

$$
\frac{-v_{3} k_{2}^{*} V_{1}^{*}-V_{2}^{*}}{\sqrt{\left(v_{3} k_{2}^{*}\right)^{2}+1}} \neq 0
$$


Involutive normal ruled surface has singular points if the curve $\alpha^{*}$ is a planar curve $k_{2}^{*}=0$ and $v_{2}=\frac{1}{k_{1}^{*}}$; under the condition

$$
-v_{2} k_{2}^{*} V_{1}^{*}+\left(1-v_{2} k_{1}^{*}\right) V_{3}^{*}=0
$$

Normal ruled surface has singular points if the curve $\alpha^{*}$ is a planar curve

$$
k_{2}^{*}=0 \Longrightarrow k_{2}^{*}=\frac{k_{2}^{\prime} k_{1}-k_{1}^{\prime} k_{2}}{(c-s) k_{1}\left(k_{1}^{2}+k_{2}^{2}\right)}=0 \Longrightarrow\left(\frac{k_{1}}{k_{2}}\right)^{\prime}=0 \Longrightarrow \frac{k_{1}}{k_{2}}=\text { constant }
$$

Hence we can say that involutive normal ruled surface has singular points if the curve $\alpha$ is a general helix with $v_{2}=\frac{1}{k_{1}^{*}}$ parameter. Also we can find distribution parameter of the involutive Frenet ruled surface of four involutive Frenet ruled surfaces along the curve $\alpha$ in terms of Frenet apparatus of curve $\alpha$

Theorem 2. The distribution parameter of the involutive Frenet ruled surface are

$P_{1}^{*}=P_{4}^{*}=0$,

$P_{2}^{*}=k_{2}^{*}=\frac{-k_{2}^{2}\left(\frac{k_{1}}{k_{2}}\right)^{\prime}}{(c-s) k_{1}\left(k_{1}^{2}+k_{2}^{2}\right)}$,

$P_{3}^{*}=k_{1}^{*}=\frac{\sqrt{k_{1}^{2}+k_{2}^{2}}}{(c-s) k_{1}}$.

Proof. From the $P_{X}=\frac{\operatorname{det}\left(\alpha^{\prime}, X, X^{\prime}\right)}{\left\langle X^{\prime}, X^{\prime}\right\rangle}$ definition of distribuiton parameter, it is trivial.

\section{Singularity and distribution parameters of Bertrandian Frenet ruled surfaces}

In this subsection first, we give the tangent, normal, binormal, Darboux Frenet ruled surfaces of the Bertran mate $\alpha^{* *}$. Further we write their parametric equations in terms of the Frenet apparatus of the Bertrand curve $\alpha$. Hence they are called collectively" Bertrandian Frenet ruled surfaces of curve $\alpha$ " as in the following way. Let $\left(\alpha, \alpha^{* *}\right)$ Bertrand curves pair and the quantities $\left\{V_{1}, V_{2}, V_{3}, \tilde{D}\right\}$ and $\left\{V_{1}^{* *}, V_{2}^{* *}, V_{3}^{* *}, \tilde{D}^{* *}\right\}$ are collectively Frenet-Serret vector of the curves $\alpha$ and the Bertrand mate $\alpha^{* *}$, respectively,

$$
\begin{array}{ll}
\varphi_{1}^{* *}\left(s, w_{1}\right)=\alpha+\lambda V_{2}+w_{1} \frac{\beta V_{1}+\lambda V_{3}}{\sqrt{\lambda^{2}+\beta^{2}}}, & \varphi_{2}^{* *}\left(s, w_{2}\right)=\alpha+\left(\lambda+w_{2}\right) V_{2}, \\
\varphi_{3}^{* *}\left(s, w_{3}\right)=\alpha+\lambda V_{2}+w_{3}\left(\frac{-\lambda V_{1}+\beta V_{3}}{\sqrt{\lambda^{2}+\beta^{2}}}\right), & \varphi_{4}^{* *}\left(s, w_{4}\right)=\alpha+\lambda V_{2}+w_{4} \frac{k_{1} \sqrt{\lambda^{2}+\beta^{2}}}{\left(\beta k_{1}-\lambda k_{2}\right)} \tilde{D}
\end{array}
$$

are the parametrization of the ruled surface which are called Bertrandian tangent ruled surface, Bertrandian normal ruled surface, Bertrandian binormal ruled surface and Bertrandian Darboux ruled surface, respectively, [5].

Theorem 3. The normal vector fields $\eta_{1}^{* *}, \eta_{2}^{* *}, \eta_{3}^{* *}$, and $\eta_{4}^{* *}$ of ruled surfaces $\varphi_{1}^{* *}, \varphi_{2}^{* *}, \varphi_{3}^{* *}$, and $\varphi_{4}^{* *}$, respectively, along the curve Bertrand mate $\alpha^{*}$, can be expressed by the following matrix;

$$
\left[\begin{array}{l}
\eta_{1}^{* *} \\
\eta_{2}^{* *} \\
\eta_{3}^{* *} \\
\eta_{4}^{* *}
\end{array}\right]=\left[\begin{array}{ccc}
0 & 0 & -1 \\
\frac{-w_{2} k_{2}^{* *}}{\sqrt{\left(v_{2} k_{2}^{*}\right)^{2}+\left(1-v_{2} k_{1}^{*}\right)^{2}}} & 0 & b_{3}^{*}=\frac{\left(1-w_{2} k_{1}^{* *}\right)}{\sqrt{\left(v_{2} k_{2}^{*}\right)^{2}+\left(1-v_{2} k_{1}^{*}\right)^{2}}} \\
\frac{-w_{2}}{\sqrt{\left(v_{3} k_{2}^{*}\right)^{2}+1}} & \frac{-1}{\sqrt{\left(v_{3} k_{2}^{*}\right)^{2}+1}} & 0 \\
0 & -1 & 0
\end{array}\right]\left[\begin{array}{c}
V_{1}^{* *} \\
V_{2}^{* *} \\
V_{3}^{* *}
\end{array}\right]
$$

Theorem 4. Bertrandian Frenet ruled surfaces, respectively, along the curve $\alpha$ has no singular points. 
Proof.Let $\eta_{1}, \eta_{2}, \eta_{3}$, and $\eta_{4}$ be the normal vector fields of involutive Frenet ruled surfaces $\varphi_{1}^{* *}, \varphi_{2}^{* *}, \varphi_{3}^{* *}$, and $\varphi_{4}^{* *}$, respectively, along the curve $\alpha$. Using the above Bertrandian tangent ruled surface has no singular points since $V_{3}^{* *}$ $\neq 0$. Bertrandian Darboux ruled surface has no singular points since $V_{2}^{* *} \neq 0$ Bertrandian binormal ruled surface has no singular points under the condition

$$
\frac{-w k_{2}^{* *} V_{1}^{* *}-V_{2}^{* *}}{\sqrt{\left(w_{3} k_{2}^{* *}\right)^{2}+1}}=0 .
$$

Bertrandian normal ruled surface has no singular points since curve $k_{2}^{*}=\frac{1}{\left(\lambda^{2}+\beta^{2}\right) k_{2}} \neq 0$ even for the parameter

$$
w_{2}=\frac{1}{k_{1}^{*}}=\frac{\left(\lambda^{2}+\beta^{2}\right) k_{2}}{\beta k_{1}-\lambda k_{2}}
$$

under the condition

$$
-w_{2} k_{2}^{* *} V_{1}^{* *}+\left(1-v_{2} k_{1}^{* *}\right) V_{3}^{*}=0
$$

Normal ruled surface has singular points if the curve $\alpha^{* *}$ is a planar curve $k_{2}^{* *}=0$. Hence we can say that normal ruled surface has singular points if the curve $\alpha$ is a general helix.

Also we can find distribution parameter of the involutive Frenet ruled surface of four Bertrandian Frenet ruled surfaces along the curve $\alpha$ in terms of Frenet apparatus of curve $\alpha$.

Theorem 5. The distribution parameter of the Bertrandian Frenet ruled surface are

$P_{1}^{* *}=P_{4}^{* *}=0$,

$P_{2}^{* *}=k_{2}^{* *}=\frac{1}{\left(\lambda^{2}+\beta^{2}\right) k_{2}}$,

$P_{3}^{* *}=k_{1}^{* *}=\frac{\beta k_{1}-\lambda k_{2}}{\left(\lambda^{2}+\beta^{2}\right) k_{2}}$.

Proof. From the $P_{X}=\frac{\operatorname{det}\left(\alpha^{\prime}, X, X^{\prime}\right)}{\left\langle X^{\prime}, X^{\prime}\right\rangle}$ definition of distribution parameter, it is trivial.

\section{Conclusion}

Since the distribution parameter $P_{1}^{*}, P_{4}^{*}, P_{1}^{* *}$ and $P_{4}^{* *}$ are zero, involutive tangent ruled surface, involutive Darboux ruled surface Bertrandian tangent ruled surface, Bertrandian Darboux ruled surface are developable surfaces. Involutive normal ruled surface is developable surface, since $P_{2}^{*}=k_{2}^{*}$, and

$$
-k_{2}^{2}\left(\frac{k_{1}}{k_{2}}\right)^{\prime}=0
$$

Involutive binormal ruled surface are not developable surface since

$$
P_{3}^{*}=k_{1}^{*} \text { and } \sqrt{k_{1}^{2}+k_{2}^{2}} \neq 0 .
$$

Bertrandian normal ruled surface is not developable surface. Bertrandian binormal ruled surface is developable surface if $k_{1}^{* *}=0$, where

$$
\lambda=\frac{k_{1}}{k_{1}^{2}+k_{2}^{2}}
$$




\section{References}

[1] Çalışkan, M. and Bilici, M., Some Characterizations for the pair of Involute-Evolute Curves in Euclidean space E $E^{3}$, Bulletin of Pure and Applied Sciences, 21(2), 289-294, 2002.

[2] Do Carmo, M. P., Differential Geometry of Curves and Surfaces, Prentice-Hall, ISBN 0-13-212589-7, 1976.

[3] Gray, A. Modern Differential Geometry of Curves and Surfaces with Mathematica, 2nd ed. Boca Raton, FL: CRC Press, 205, 1997.

[4] Hacısalihoğlu H.H., Differential Geometry(in Turkish), vol.1, İnönü Üniversity Publications, 1994.

[5] Kılıçoğlu Ş, Şenyurt, S. and Hacısalihoğlu H.H., On the striction curves of Involute and Bertrandian Frenet ruled surfaces in $\mathbb{E}^{3}$, Applied Mathematical Sciences, 9(142), 7081 - 7094, 2015, http://dx.doi.org/10.12988/ams.2015.59606.

[6] Şenyurt, S., Kılıçoğlu Ş. and Hacısalihoğlu H.H., An Examination on the Positions of Frenet Ruled Surfaces Along Bertrand Pairs $\alpha$ and $\alpha^{*}$ According to Their Normal Vector Fields in $E^{3}$, Applied Mathematical Sciences, 9(142), 7095 - 7103, 2015.

[7] Izumiya, S., Takeuchi, N., Special curves and Ruled surfaces, Beitrage zur Algebra und Geometrie Contributions to Algebra and Geometry,44(1), 203-212, 2003.

[8] Schief W., On the integrability of Bertrand curves and Razzaboni surfaces. Journal of Geometry and Physics, Volume 45, Issues 1-2, Pages 130-150, February, 2003.

[9] Şenyurt, S. and K1lıçoğlu Ş., On the differential geometric elements of the involute D̃ scroll, Adv. Appl. Cliff ord Algebras 2015 Springer Basel,doi:10.1007/s00006-015-0535-z. 